\title{
Apolipoprotein J/clusterin limits the severity of murine autoimmune myocarditis
}

\author{
Lea McLaughlin, ${ }^{1}$ Guang Zhu, ${ }^{2}$ Meenakshi Mistry, ${ }^{1}$ Cathy Ley-Ebert,${ }^{2}$ William D. Stuart,${ }^{1}$ \\ Carolyn J. Florio, ${ }^{2}$ Pamela A. Groen, ${ }^{3}$ Sandra A. Witt, ${ }^{4}$ Thomas R. Kimball, ${ }^{4}$ \\ David P. Witte, ${ }^{3}$ Judith A.K. Harmony, ${ }^{1}$ and Bruce J. Aronow ${ }^{2}$
}

${ }^{1}$ Department of Pharmacology and Cell Biophysics, University of Cincinnati College of Medicine, Cincinnati, Ohio, USA
2Division of Molecular and Developmental Biology,
${ }^{3}$ Department of Pathology and Laboratory Medicine, and
${ }^{4}$ Division of Molecular Cardiovascular Biology, Children's Hospital Research Foundation, Cincinnati, Ohio, USA

Address correspondence to: Judith A.K. Harmony, Department of Pharmacology and Cell Biophysics, University of Cincinnati College of Medicine, PO Box 670575, Cincinnati, Ohio 45267-0575, USA.

Phone: (513) 558-4723; Fax: (513) 558-1169; E-mail: jharmony@fuse.net.

Received for publication December 2, 1999, and accepted in revised form September 5, 2000.

\begin{abstract}
Apolipoprotein $\mathrm{J} /$ clusterin (apoJ/clusterin), an intriguing protein with unknown function, is induced in myocarditis and numerous other inflammatory injuries. To test its ability to modify myosin-induced autoimmune myocarditis, we generated apoJ-deficient mice. ApoJ-deficient and wild-type mice exhibited similar initial onset of myocarditis, as evidenced by the induction of two early markers of the T cell-mediated immune response, MHC-II and TNF receptor p55. Furthermore, autoantibodies against the primary antigen cardiac myosin were induced to the same extent. Although the same proportion of challenged animals exhibited some degree of inflammatory infiltrate, inflammation was more severe in apoJ-deficient animals. Inflammatory lesions were more diffuse and extensive in apoJ-deficient mice, particularly in females. In marked contrast to wild-type animals, the development of a strong generalized secondary response against cardiac antigens in apoJ-deficient mice was predictive of severe myocarditis. Wild-type mice with a strong $\mathrm{Ab}$ response to secondary antigens appeared to be protected from severe inflammation. After resolution of inflammation, apoJ-deficient, but not wild-type, mice exhibited cardiac function impairment and severe myocardial scarring. These results suggest that apoJ limits progression of autoimmune myocarditis and protects the heart from postinflammatory tissue destruction.
\end{abstract}

J. Clin. Invest. 106:1105-1113 (2000).

\section{Introduction}

Autoimmunity, a frequent cause of morbidity and mortality in children and adults, can cause disease in a wide variety of organ systems (1). Myocarditis is a clinically severe inflammatory disease (2) characterized by the presence of injured or necrotic myocytes engaged with inflammatory cells adjacent to apparently healthy myocytes. Autoimmunity is thought to play a major role in the development or progression of myocarditis in humans $(3,4)$, which can result in dilated cardiomyopathy or death (5). Initiating agents include both noninfectious and infectious agents, such as drugs, viruses, bacteria, and protozoa (6).

Animal models have established that host immune responses can cause myocardial damage during myocarditis through cytokines, nitric oxide (7), and activated macrophage-generated free radicals (8). In both clinical and experimental myocarditis, autoimmunity is accompanied by robust production of autoantibodies to cardiac antigens, notably myosin heavy chain, adenine nucleotide translocator, and cardiac sarcoplasmic reticulum calcium ATPase (6). Different mouse strains have indicated that multiple mechanisms govern myocarditis susceptibility and severity. C57BL/6 mice are relatively resistant to autoimmune myocarditis. DBA/2 mice develop myocarditis through humoral immunity, whereas the $T$ cell-mediated immune response predominates in $\mathrm{BALB} / \mathrm{c}$ mice (9-11). In A/J mice, both immune pathways contribute (9). This suggests that susceptibility to disease initiation and mechanism of disease progression are determined by the interplay of multiple genes.

Both cardiac myosin and viral infection models of myocarditis $(12,13)$ have been combined with transgenic and gene ablation strategies to facilitate dissection of pathways that contribute to inflammatory tissue injury. Critical roles have been established for cytokines and their receptors, e.g., TNFrp55, IL-1, CD4, CD8, CD28, CD45, and tyrosine kinase p56 1ck (13-17). In addition, diverse proteins are modulated during the development of myocarditis, including fibrin and fibronectin (18), perforin (19), $\beta$-globin (20), and apolipoprotein J/clusterin (apoJ) (21). However, it is not yet clear which of these modulates the development of pathology or merely serves as a bioindicator of disease progression. It is possible that distinct 
classes of proteins delimit the extent or severity of immune-mediated tissue damage by their action at initiation or progression stages.

Based on its expression pattern, inducibility, and biochemical activities (22-25), we have hypothesized that apoJ functions to protect against inflammatory injury. In the normal adult mouse and rat heart, apoJ is constitutively expressed in atrial, but not ventricular, myocytes, yet is markedly upregulated in ventricular myocytes situated at dynamic interfaces between healthy and injured myocardium $(21,26)$. It also exhibits strong induction in numerous other pathologic processes in diverse cell types and tissues (27-33). ApoJ has been linked to the immune system based on its ability to bind immunoglobulin and complement in vitro, but the physiologic consequences of these activities have been controversial (34).

We generated apoJ-null mice, found minimal phenotype in unperturbed animals, and have therefore sought to elucidate a role for apoJ in myosin-induced autoimmune myocarditis, an important autoimmune inflammatory disease model. The severity of autoimmune myocarditis was significantly altered compared with wild-type animals. Both humoral or cell-mediated immune responses appeared to initiate normally, but the extent of the inflammatory damage was considerably more severe in the deficient animals, and this led to significant long-term loss of cardiac function. These results provide insight into the in vivo function of apoJ and suggest the involvement of apoJ in pathways that limit the progression of autoimmune disease and inflammatory injury.

\section{Methods}

Preparation of the targeting construct and generation of mutant mice. A genomic clone containing the mouse apoJ gene was isolated from a strain $129 / \mathrm{SvJ}$ phage library (35) and completely sequenced (accession no. AF182509). The targeting vector, pApoJ-KO3, was constructed in pBluescriptII with restriction fragments that included a 7.2-kb BsaHI-EcoRI long-homology restriction fragment that contained exons 3-7, the HPRT cassette with the mouse PGK promoter, and a EcoRI-AgeI short-homology fragment from the $5^{\prime}$ flanking portion of the apoJ gene. A PGK-TK cassette was present upstream of the short-homology fragment. The orientation of the HPRT gene was the same as that of the apoJ gene. Electroporation and selection of targeted embryonic stem (ES) cells, blastocyst-mediated transgenesis, and mating of chimeric mice with Swiss Black mice was carried out as described previously (35).

ApoJ-deficient mice were first generated in the Swiss Black outbred genetic background and subsequently backcrossed for seven generations into myocarditissusceptible FVB/N mice. ApoJ-deficient and wild-type $\mathrm{FVB} / \mathrm{N}$ mice used in all experiments were obtained from the mating of heterozygous $\mathrm{apoJ}^{+/-}$animals.

Genotypic analysis of apoJ gene-targeted mice. DNA from ES cell clones and tail biopsies was analyzed by Southern hybridization, using probes from outside the homology regions used to prepare the construct. The $3^{\prime}$ probe was a 0.6-kb EcoRI-NsiI fragment containing exon 8 . The $5^{\prime}$ probe was a $0.4-\mathrm{kb}$ PstI-EcoRI fragment, beginning $1 \mathrm{~kb}$ upstream of the transcription start site. Genotyping was also performed by PCR analysis, using three primers that amplified both wild-type and mutant alleles in the same reaction. These were: SHF-3 (5'-ACGATGTGGAAGGATGTGGAAGATGAACATG-3') forward primer, recognizing both mutant and wild-type alleles and corresponding to genomic sequences near the $5^{\prime}$ end of the short homology fragment; JKO-R2 (5'TGGTGATGGGGCTCTAGTCACCTCCCACTTC-3'), reverse wild-type primer complementary to sequences just $3^{\prime}$ to the deleted first exon; and PGK-2 (5'-CTGCTAAAGCGCATGCTCCAGACTGCCTTG-3'), reverse knockout primer corresponding to sequences in the promoter region of the HPRT cassette.

PCR for wild-type (500 bp) and mutant (540 bp) alleles was performed with cycles consisting of 3 minutes at $94^{\circ} \mathrm{C}, 45$ seconds at $52^{\circ} \mathrm{C}$, and 45 seconds at $72^{\circ} \mathrm{C}$.

Myocarditis. Cardiac myosin from FVB/N mice was purified (36), emulsified in CFA (Sigma Chemical Co., St. Louis, Missouri, USA), and $100 \mu \mathrm{g}$ injected subcutaneously to initiate autoimmune myocarditis $(12,21)$. Recipient mice were primed intraperitoneally with 500 ng of pertussis toxin (List Biological Labs, Campbell, California, USA). One week later, a second $100-\mu \mathrm{g}$ injection of myosin in incomplete Freund's adjuvant (IFA; Sigma Chemical Co.) was administered. Control mice were injected with pertussis toxin in CFA and subsequently with IFA.

Histology. Tissues for immunohistochemistry were flash frozen in OCT (Miles Laboratories, Elkhart, Indiana, USA). Tissues for in situ hybridization and histological evaluation were fixed in $4 \%$ (wt/vol) paraformaldehyde, as described (26). In situ hybridization analysis was as previously reported (37). Heart tissues were evaluated histologically for inflammation and damage. For each mouse, approximately 15 cryostat sections $(7 \mu \mathrm{m})$, encompassing approximately 700 $\mu \mathrm{m}$ of ventricle, were stained with hematoxylin and eosin (H\&E). The percentage of tissue that was actively involved in an inflammatory response or that showed evidence of damage was based on the average of all sections (21).

Cardiac scarring was evaluated with Masson's trichrome stain. For detection of TNFrp55, $7 \mu \mathrm{m} \mathrm{sec-}$ tions were dehydrated with acetone (10 minutes), blocked (45 minutes at room temperature) with $10 \%$ normal goat serum, incubated overnight $\left(4^{\circ} \mathrm{C}\right)$ with 10 $\mu \mathrm{g} / \mathrm{ml}$ of rabbit $\mathrm{Ab}$ against mouse $\mathrm{TNF}$ alpha receptor (TNFrp55; BIODESIGN International, Kennebunk, Maine, USA), and washed in buffer containing $5 \%$ normal goat serum. Immunoreactivity was detected with fluorescein-conjugated goat anti-rabbit Ab (DAKO Corp., Carpinteria, California, USA), and diluted 1:250 (30 minutes at room temperature), with propidium iodide counterstain. MHC-II expression was detected with an anti-mouse $\mathrm{I}-\mathrm{A}^{\mathrm{q}}$ biotinylated $\mathrm{Ab}$ with the 
Figure 1

Mouse apol gene and construct used for targeted knockout. The targeting vector used the HPRT/TK selection system. A short-homology fragment derived from apoJ gene sequences upstream of the promoter, and a long homology fragment from downstream apoJ gene exons. KO, knockout.

mouse anti-mouse (MOM) immunodetection and 3,3diaminobenzidine (DAB) substrate kits (Vector Laboratories, Burlingame, California, USA).

Statistical analyses. Differences between experimental groups were determined by Mann-Whitney nonparametric statistical analysis (21) and exact Wilcoxon rank sum tests using SAS (SAS Institute Inc., Cary, North Carolina, USA). StatMate (Prism Graph Pad Software Inc., http://www.sxst.it/grp_prs.htm) was used for power analysis to determine that sufficient numbers of mice had been used in each experimental group.

Western blot analysis. Absence of apoJ in knockout animals was confirmed in serum, hearts, and livers. Tissues were homogenized in RIPA buffer (Upstate Biochemicals, Lake Placid, New York, USA) and subjected to PAGE. ApoJ was detected with rabbit anti-rat apoJ Ab (38) and visualized by enhanced chemiluminescence (ECL; Amersham, Piscataway, New Jersey, USA).

Humoral autoimmune response. To evaluate the spectrum of cardiac autoantibodies elicited during myocarditis, serum from each experimental mouse was used to probe strips of PVDF membranes onto which normal heart proteins from a wild-type FVB/N heart had been blotted after electrophoresis. To do this, extract was made from hearts that were frozen, pulverized, and homogenized at pH 7.6 in Perks' lysis buffer, as described (39). Homogenates were boiled in $1 \times$ Laemmli buffer with $\beta$-mercaptoethanol and subjected to $5-10 \%$ gradient SDS-PAGE (40). Serum samples from individual mice were diluted 1:3000. Visualization used a secondary goat anti-mouse IgG Ab coupled to HRP and the ECL kit. In addition, antimyosin titer was determined by ELISA $(12,41)$. Test sera were diluted 1:20 to 1:100,000, and autoantibodies were detected with peroxidase-labeled anti-mouse IgG and IgM (ICN Radiochemicals, Irvine, California, USA) (12). The mean values plus or minus 2 SD obtained from each experimental group were compared by the two-tailed $\chi$ square test for independent populations.

Echocardiography. Echocardiography was performed on apoJ-deficient $(n=3)$ and wild-type $(n=4) \mathrm{FVB} / \mathrm{N}$ mice 7 weeks after the initial myosin injections, as described (42), using a Hewlett Packard Sonos 2500 ultrasound system
$0 \quad 5000+10000$
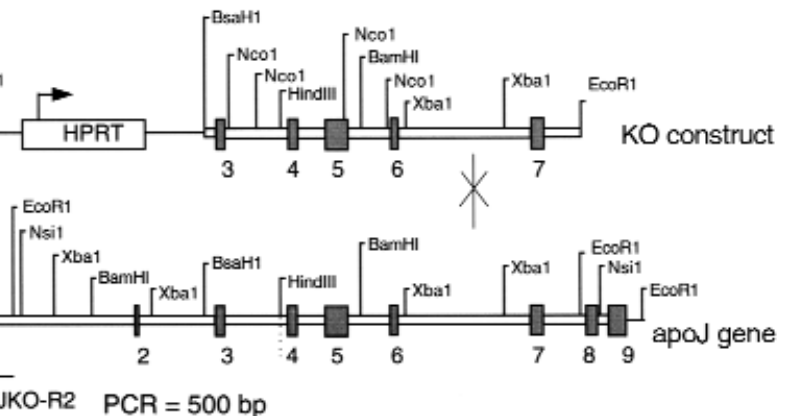
Palo Alto, California, USA). Best resolution and placement of the heart in the midfield of the ultrasound sector was obtained by fitting the transducer with an acoustic gel-filled latex balloon. This was applied to the shaved areas to create a $1-\mathrm{cm}$ standoff between the chest wall and transducer face with a depth setting of 3 or $4 \mathrm{~cm}$. Two-dimensional guided $\mathrm{M}$-mode echocardiograms were recorded to measure left ventricle end-diastolic and endsystolic dimensions (LVED, LVES), right ventricle (RV) and septal thickness. Left ventricle (LV) performance was determined by calculating the shortening fraction (SF) $(\mathrm{SF}=[\mathrm{LVED}-\mathrm{LVES}] / \mathrm{LVED})$. Data are shown as mean plus or minus standard deviation. Statistical analysis was performed by Student's $t$ test.


Figure 2

Disruption of the apoJ gene locus and loss of apoJ protein in targeted mice. (a) PCR analyses of tail DNA from progeny mice derived from mating heterozygous apoJ-deficient mice. (b) Western blot analysis of plasma and liver extracts from apoJ-deficient (KO) and wild-type (WT) mice with rabbit anti-rat apoJ polyclonal antiserum. 
Figure 3

ApoJ-deficient mice develop diffuse and severe inflammatory lesions during myocarditis. Shown are representative H\&E sections of wild-type and apoJ-deficient diseased hearts 3 weeks after initiation of myocarditis. (a, c) Wild-type animals develop focal inflammatory lesions in interstitial spaces with occasional zones of myocyte necrosis. (b-d) ApoJ deficiency results in severe and widespread inflammation, affecting large regions of the ventricle. $\mathbf{a}$ and $\mathbf{c}, \times 10$; b and $\mathbf{d}, \times 40$.

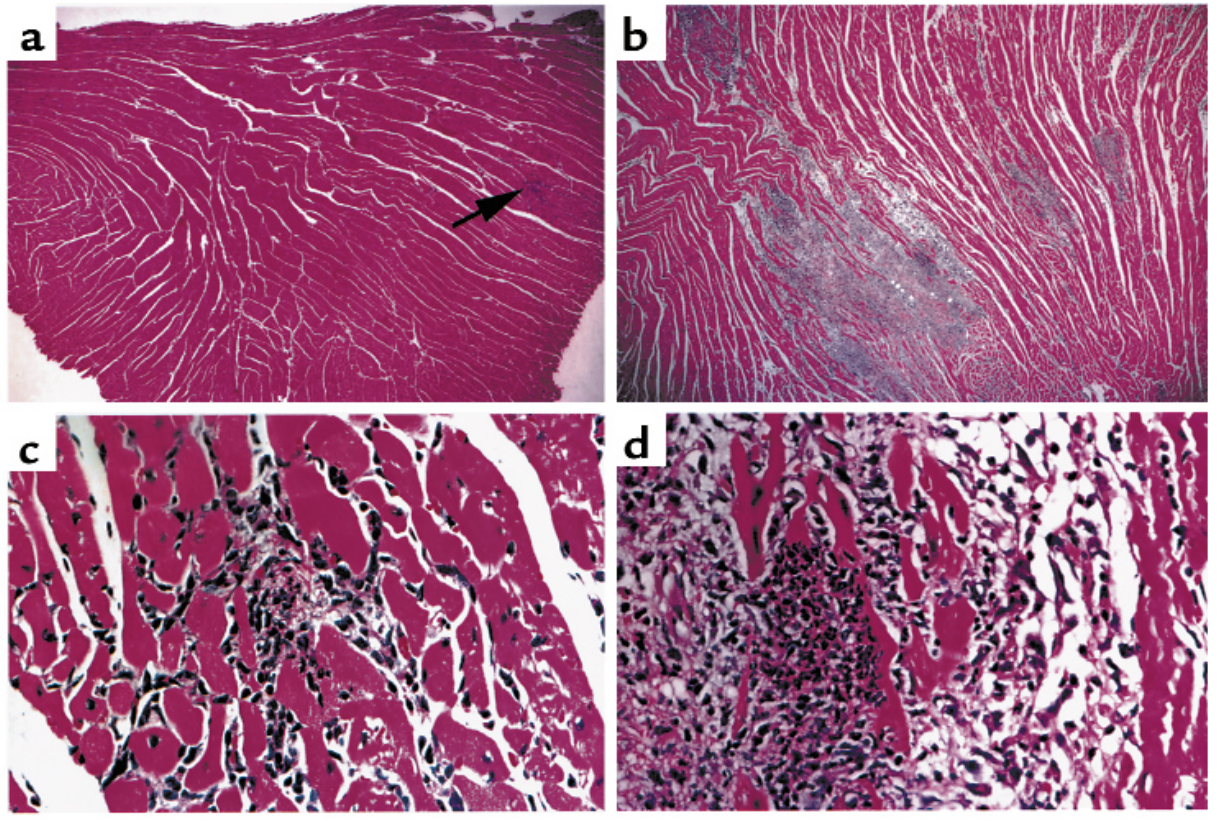

\section{Results}

ApoJ/clusterin-deficient mice. ApoJ-null mutant mice were developed by standard techniques of homologous recombination (Figure 1), using the HPRT/TK selection method (35) and confirmed by Southern analyses (data not shown), PCR, and Western blot analysis (Figure 2, $a$ and $b$ ). In addition, no apoJ mRNA was detectable in multiple mouse tissues with a full-length cDNA probe (not shown). All apoJ-deficient mice were phenotypically normal. Hearts and other organs from young adult apoJ-deficient mice were also normal by morphologic and H\&E-staining criteria. Both male and female homozygous deficient animals were fertile while young, and females gave birth to normal-size litters. Genotype analyses of several hundred FVB/N and Swiss Black weanlings from hemizygote $\times$ hemizygote matings revealed a small, but statistically significant, bias in favor of apoJ-deficient animals.

ApoJ/clusterin-deficient mice develop severe myocarditis. When immunized with cardiac myosin, virtually all wildtype and apoJ-deficient animals (100\% vs. $89 \%$, respectively) developed inflammatory heart infiltrates at 21 days. Thus, disease prevalence was not altered by apoJ deficiency. However, the severity of autoimmune myocarditis differed between the two genotypes. Wildtype mouse hearts were rarely enlarged and generally exhibited only a few small areas of pale tissue. In contrast, most hearts of apoJ-deficient mice were pale and enlarged. Typical lesions in wild-type myocardium consisted of inflammatory foci in interstitial spaces, with occasional zones of myocyte necrosis (Figure 3, a and c). ApoJ-deficient mice exhibited severe, diffuse inflammation, often associated with myocyte necrosis, edema, and large inflammatory aggregates (Figure 3, b and d).

To quantify the impact of apoJ deficiency on autoimmune myocarditis, we performed systematic serial sec- tion analyses of hearts from each of 20 apoJ-deficient and 27 wild-type mice. Overall, apoJ-deficient mice developed almost three times $(P<0.0001)$ more cardiac tissue injury (mean, 56\%) than wild-type animals (mean, 20\%) when evaluated 21 days after initiation of myocarditis (Figure 4). Ventricular damage in apoJdeficient females was sixfold greater than in wild-type females (mean of $67 \%$ vs. $11 \%$, respectively; $P<0.0001$ ). The difference in extent of cardiac injury was not as significant in males (mean, $42 \%$ vs. $29 \%$; $P<0.233$ ). Consistent with our previous results (43), there was no statistically significant sex difference in the severity of

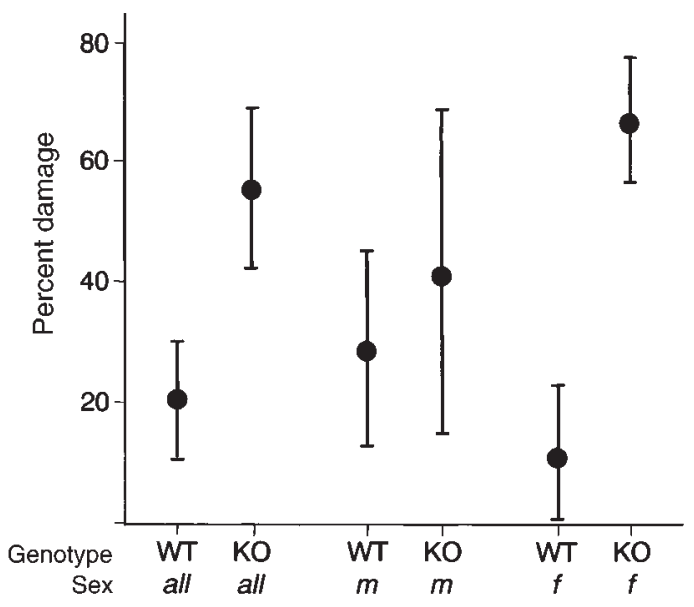

Figure 4

Increased myocarditis severity in apoJ-deficient mice. The results of three independent experiments $(\mathrm{WT}=27 ; \mathrm{KO}=20)$ were pooled and shown as the $95 \%$ confidence intervals of the mean. The damaged area of each heart was determined as the percentage of total area from 15 serial sections, encompassing over $7 \mathrm{~mm}$ across the heart. No inflammatory infiltrates were observed in control mice $(n=6)$. 


\section{Figure 5}

Myocarditis induces apoJ mRNA in wild-type, but not apoJdeficient, mice. In situ hybridization analysis of comparable severely diseased hearts of wild-type (a) and apoJ-deficient (b) mice. (c-f) Corresponding bright-field views of sections shown in $\mathbf{a}$ and $\mathbf{b}$. ApoJ mRNA is induced to high levels in wild-type mice in ventricular myofibrils surrounding lesions, specifically in myocytes adjacent to inflammatory cells (arrow) (e). No apoJ is expressed in hearts of apoJ-deficient animals. a-d, $\times 10 ; \mathbf{e}, \mathbf{f}, \times 40$.

myocarditis in wild-type $\mathrm{FVB} / \mathrm{N}$ animals $(P<0.068)$. Using nonparametric analysis, significance requires a $P$ value of less than 0.05 .

To confirm that the introduced mutation ablated expression of all forms of the apoJ transcript under injury and induction conditions, we performed in situ hybridization analysis on comparable highly inflamed hearts of wild-type (Figure 5, c and e) and knockout (Figure 5, d and $\mathrm{f}$ ) animals. The entire apoJ cDNA was used as probe. As shown previously (21), the apoJ gene was strongly induced in ventricular myocytes in diseased hearts of wild-type mice (Figure 5a) adjacent to the regions of inflammation (arrow, Figure 5e). No induced expression of apoJ or apoJ-like mRNA was observed in apoJ-deficient animals (Figure 5b), indicating that to the limit of detection, no portion of the apoJ gene was expressed by the engineered mice.

Early markers of autoimmune myocarditis are not altered in apoJ/clusterin-deficient mice. Tissue damage during autoimmune myocarditis can occur as a result of humoral and/or T cell-mediated immune pathways. Since the relative contribution of these immune pathways to myosin-induced myocarditis in $\mathrm{FVB} / \mathrm{N}$ mice is unknown, we assessed markers that indicate activation of both immune pathways. MHC-II and TNFrp55 are activated early in cell-mediated immunity. TNF, a proinflammatory cytokine produced by

\section{Figure 6}

Induction of cardiac MHC-II is similar in apoJdeficient and wild-type mice. MHC-II is induced the hearts of both (a) wild-type and (b) apoJdeficient mice. Cryostat sections were probed with a mouse anti-mouse MHC-II Ab and detected with DAB. No antigen was detected in hearts of wild-type or apoJ-deficient mice (c) when secondary Ab was omitted, or (d) when control mice were injected with CFA alone (sections from apoJ-deficient animals shown). $\times 20$.
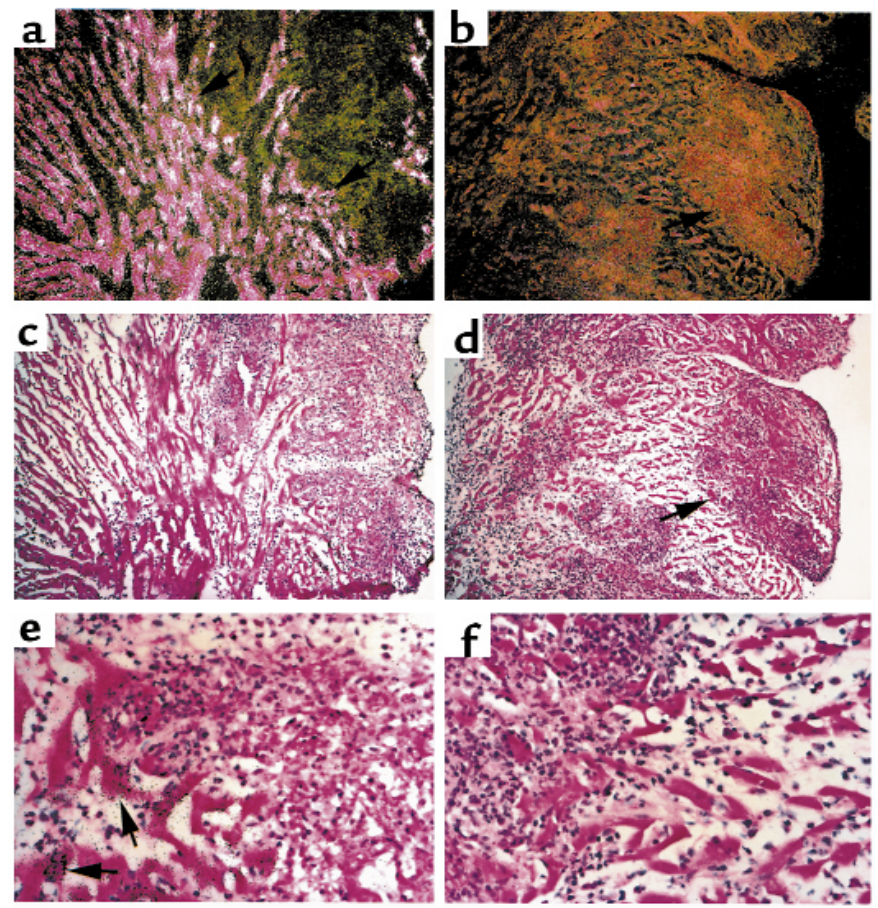

macrophages and myocytes, has been specifically implicated in cardiovascular disease (44-46). The humoral response was evaluated by production of Ab's against cardiac antigens, including myosin.

Tissues from apoJ-deficient and wild-type mice, selected for both moderate and severe myocardial injury (15\% and $70 \%$ ), were evaluated for TNFrp55 and MHC-II expression. MHC-II was induced to the same extent in animals of both genotypes in inflammatory lesions and within the interstitial cell population defined by Pummerer et al. (15) (Figure 6). Inflammatory cells in the myocardium of wild-type
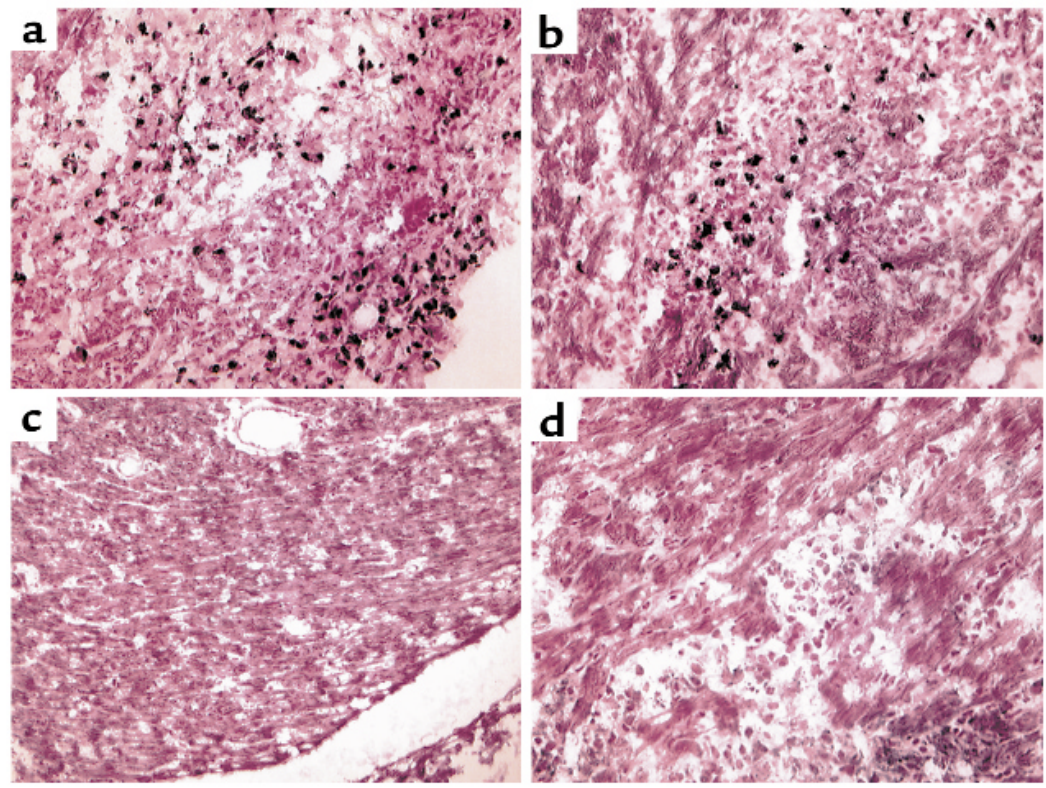

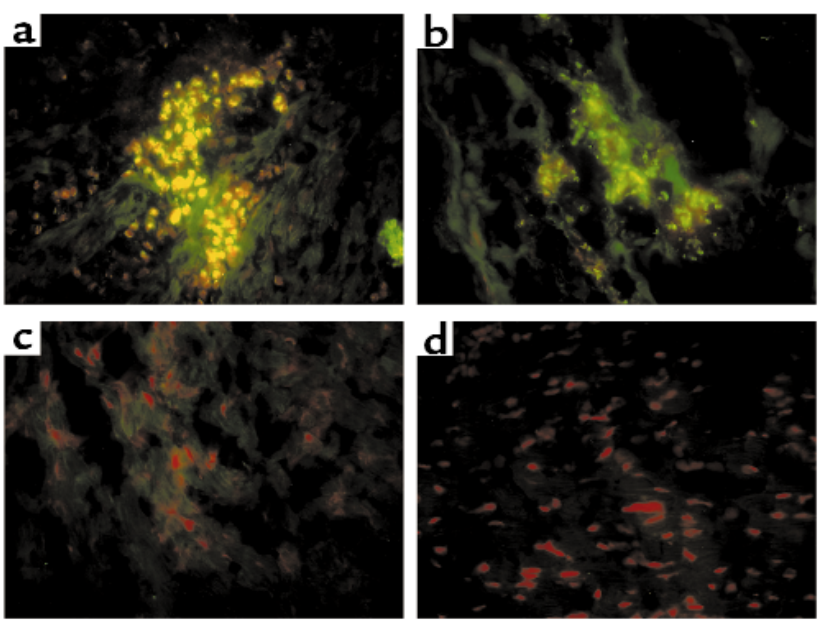

Figure 7

TNFrp55 is similarly induced in both genotypes. TNFrp55 is induced in hearts of both wild-type (a) and (b) apoJ-deficient mice. Cryostat sections were probed with a rabbit anti-mouse TNFrp55 Ab and detected with fluorescein-labeled anti-rabbit Ab. No antigen was detected in hearts of control (c) wild-type or (d) apoJ-deficient mice. $\times 40$.

and apoJ-deficient mice also expressed similar TNFrp55 reactivity (Figure 7). MHC-II and TNFrp55 were undetectable in hearts of control mice.

Serum antimyosin Ig (IgG plus IgM) autoantibody titers were determined by ELISA (10). ApoJ-deficient animals $(n=8)$ exhibited a threefold higher severity of myocarditis $(P<0.005)$ compared with wild-types $(n=13)$. However, antimyosin titers did not differ: wild-type $=4,600 \pm 2,700$ vs. knockout $=5,600 \pm$ 2,700 (mean values $\pm 2 \mathrm{SD} ; P<0.2$ ).

As a possible explanation for increased inflammatory severity in apoJ-deficient mice, we questioned whether the $\mathrm{Ab}$ response against the inflamed hearts was more generalized in the absence of apoJ. To test this, sera from affected mice were used as primary $\mathrm{Ab}$ to detect antigens present in normal heart. As shown by Western blot analysis (Figure 8), most sera exhibited significant $\mathrm{Ab}$ reactivity against multiple cardiac protein bands. In wild-type animals, generalized immunoreactivity was inversely associated with inflammatory disease severity. In other words, wildtype animals with low generalized

\section{Figure 8}

Secondary autoantibody responses in apoJ-deficient (KO) and wild-type (WT) mice. Sera collected 21 days after mice were inoculated with cardiac myosin were used as a source of Ab's in a Western blot analysis of antigens in normal heart homogenate, resolved by electrophoresis. All sera were diluted 1:1000. My, myosin. immunoreactivity tended to have severe myocarditis, whereas those with strongest generalized immunoreactivity exhibited the least severe disease. In contrast, apoJdeficient animals with strong autoantibody response all exhibited extensive lesions.

Postmyocarditis heart dysfunction in apoJ/clusterin-deficient mice. We used M-mode echocardiography to determine if apoJ deficiency has long-term consequences for heart function after myocarditis. Parameters of left-ventricle geometry and performance were measured, including heart rate and shortening fraction, 7 weeks after inflammatory injury (Table 1 and data not shown). Wild-type mice did not exhibit diminished heart function compared with control mice of both genotypes without myocarditis. In contrast, based on shortening fraction, cardiac function in apoJ-deficient mice was significantly impaired compared with their wild-type counterparts $(P<0.05)$.

Since decreased shortening fraction implies loss of myocardial elasticity or compliance, we evaluated the heart tissue architecture (Figure 9). Four mice (two males and two females) from each genotype were given 7 weeks to recover from myocarditis. One female knockout mouse died just before analysis. In the apoJ-deficient mice, there was widespread loss of myocardial fibers, which were replaced by trichromepositive collagen. Wild-type mice, however, exhibited regular myocardium with only limited and small areas of collagen. No inflammatory cells remained in hearts from either genotype.

\section{Discussion}

Our results indicate that apoJ provides a protective role in autoimmune myocarditis. Deficiency of apoJ affected three parameters of myocarditis. The first was the severity of acute myocardial inflammation, which was significantly greater in apoJ-deficient animals. The second was the relationship between titer of

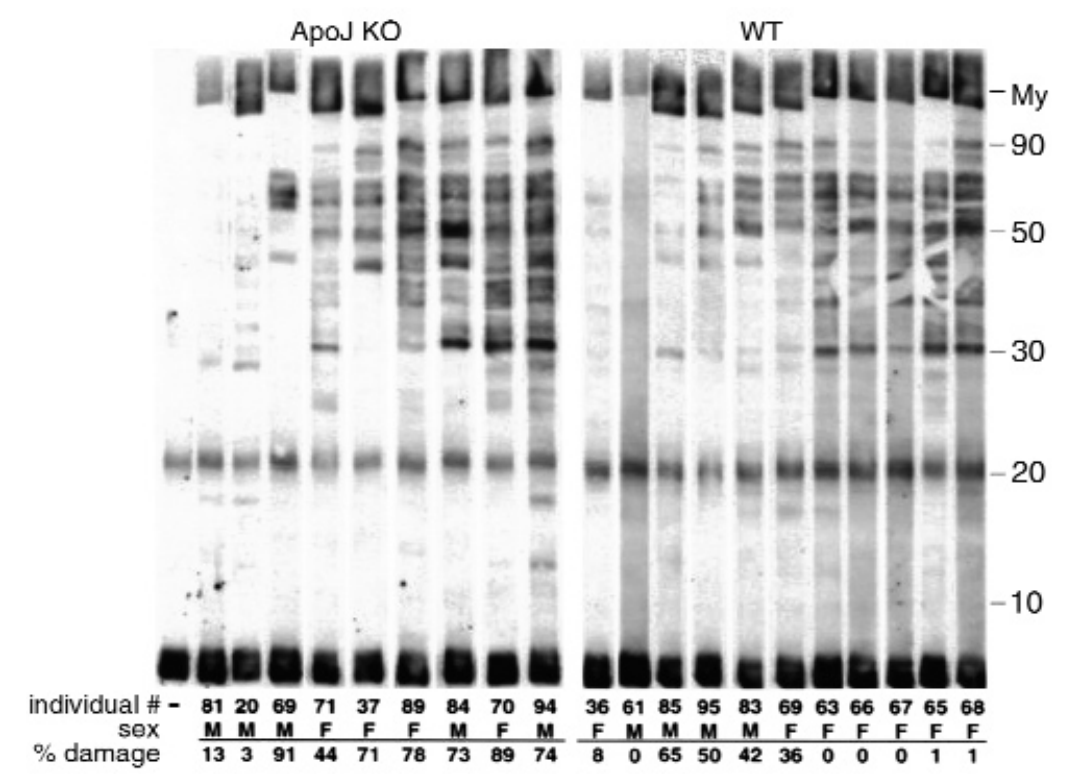


secondary Ab's against cardiac antigens and disease severity. Secondary Ab reactivity was inversely associated with the extent of inflammation in wild-type animals, but positively associated in apoJ-deficient mice. The third was the progression of the acute inflammation to myocardial scarring, which was greater in apoJ-deficient mice. Not affected was the prevalence or occurrence frequency of detectable myocarditis, which was similar in both genotypes. Also not affected by apoJ deficiency were several indicators of early stages in myocarditis, including cardiac edema, infiltration of monocytic inflammatory cells, antimyosin $\mathrm{Ab}$ titers, and induction of MHC-II and TNF receptor expression. Thus, that most wild-type mice developed some myocarditis, but with generally low severity, suggests the hypothesis that apoJ regulates disease progression after the initiated stage.

Female mice were the most sensitive to apoJ deficien$c y$, based largely on the low severity scores of the wildtype females. ApoJ-deficient males were less prone to the most severe myocarditis at 21 days, but did exhibit compromised cardiac function and evidence of postinflammatory pan-cardiac fibrosis at 7 weeks. Although myocarditis in wild-type FVB/N mice does not appear to depend significantly on sex (43), males of other strains (Balb/C, C57BL/6) exhibit increased severity (47). Our studies show a trend in that direction, but not at the level that exceeds statistical significance.

Sex-associated variation has been reported in immune mechanisms responsible for myocarditis in mice. In Coxsackievirus B3-mediated myocarditis, which appears very similar to cardiac myosin-based disease at the acute stage, there is a sex-associated reversal in the Th-cell response. Th1 cells, predominant in the response of male mice, produce mainly IL-2, IFN- $\gamma$, and TNF, and are primarily responsible for cell-mediated immunity. In contrast, Th2 cells predominate during the female immune response and produce IL-4, IL-5, and IL-10, involved in humoral immunity. Sex hormones have been implicated to modify the relative production or function of Th1 and Th2 cells derived from their common Th0 cell precursor $(48,49)$. Thus, a possible explanation for sex differences in FVB/N mice is that apoJ alters the balance between the execution of Th1 and Th2 pathways.

The humoral response is a significant component of myocarditis in humans and in animal models (50, 51), but its relationship to tissue injury is unclear. Both wild-type and apoJ-deficient mice developed similar and robust primary antimyosin $\mathrm{Ab}$ response, as well as frequent occurrence of $\mathrm{Ab}$ responses against other cardiac antigens. Among wild-type mice that developed a strong secondary humoral response, most developed little myocarditis. In contrast, among apoJ-deficient mice that had high secondary immunoreactivity, myocarditis was severe in both males and females. Thus, the presence of apoJ protected against the development of severe tissue injury in the presence of significant secondary autoanti-
Table 1

Cardiac function is impaired in apoj-deficient mice compared with wild-type animals 7 weeks after autoimmune myocarditis

\begin{tabular}{|c|c|c|c|c|}
\hline & $\begin{array}{l}\text { ApoJ } J^{-/-} \\
\text {control } \\
(n=5)\end{array}$ & $\begin{array}{l}\text { Wild-type } \\
\text { control } \\
\quad(n=6)\end{array}$ & $\begin{array}{c}\text { Apof }^{-/-} \\
\text {with } \\
\text { myocarditis } \\
(n=3)\end{array}$ & $\begin{array}{l}\text { Wild-type } \\
\text { with } \\
\text { myocarditis } \\
(n=4)\end{array}$ \\
\hline & $23 \pm 2.5$ & $27 \pm 2$ & 29 & $23 \pm 4.7$ \\
\hline - rt woigh & 024 & $0.31 \pm$ & & $0.2 \pm 0.1$ \\
\hline & $.4 \pm$ & 51.7 & $35.1 \pm 4.6^{A}$ & $60 \pm 11.9$ \\
\hline
\end{tabular}

Calculation of the SF is described in Methods. One female apof-1- died at 6.5 weeks after myocarditis. ${ }^{A} A$ significant decrease $(P<0.05)$ in the SF indicates functional impairment.

bodies. The simplest interpretation of these results is that apoJ plays a role in the suppression of immune complex-mediated cardiac pathology.

The protective effect of apoJ against acute myocarditis prevented subsequent heart function impairment. This effect appeared to be independent of sex. Analyzed after 7 weeks, apoJ-deficient mice exhibited markedly decreased shortening fraction as determined by echocardiography, consistent with their high level of myocyte loss and cardiac scarring. In contrast, wildtype mice exhibited no cardiac functional impairment and only limited scarring.

How does apoJ exert protective effects, and do its actions suggest important avenues for further analysis of autoimmune modifiers? To limit progression of
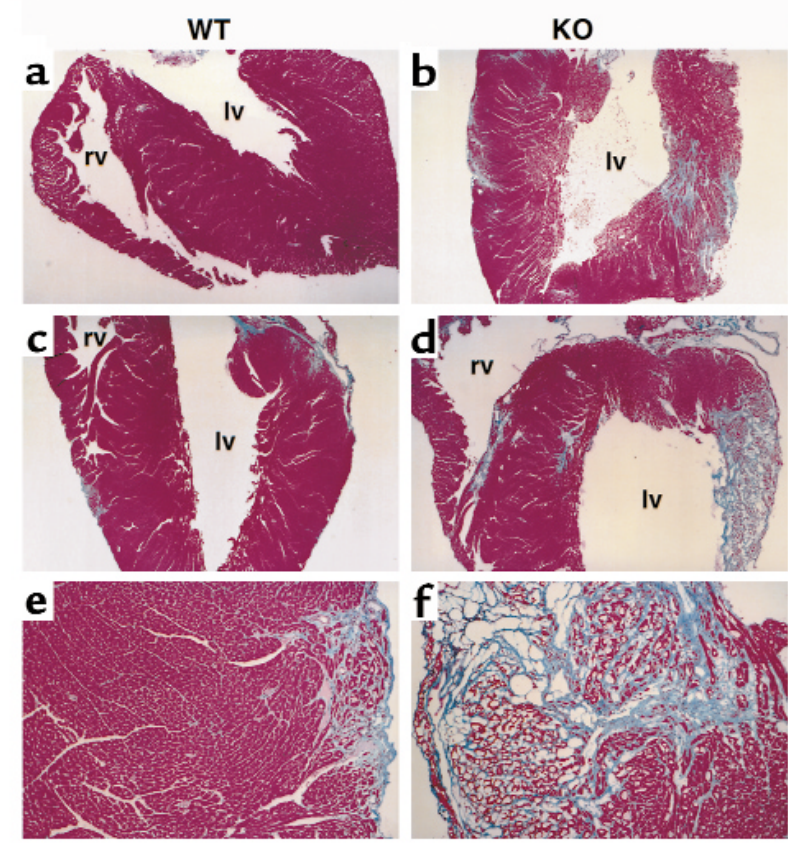

\section{Figure 9}

Severe myocardial scarring in apoj-deficient hearts after myocarditis. Representative sections from (a, c, e) wild-type (WT) and (b, d, f) apoJ-deficient ( $\mathrm{KO})$ mice subjected to echocardiography were stained with Masson's trichrome. Normal cardiac myofibrils stain red/purple and collagen deposits stain light blue. Iv, left ventricle; rv, right ventricle. a-d, $\times 4$; e, f, $\times 20$. 
inflammatory injury, apoJ may function at the level of the cardiomyocyte or by modulating the immune effectors. This could be accomplished by many mechanisms. Given the broad spectrum of biomolecules that have been reported to bind apoJ, it is possible that cardiomyocytes coated with or producing apoJ are resistant to attack by immune complexes or effector cells. Alternatively, apoJ may moderate the activation or action of immune effector components in the setting of an initiated autoimmune response. ApoJ has been suggested to act as an extracellular mediator of TNF cytotoxicity (52-54), for example. A third possibility is that apoJ aids in the disposal of the products of cell death or immune complexes, effectively preventing the continued activation or recruitment of immune effector cells. In addition, apoJ has also been shown to accumulate in cell nuclei under some conditions $(55,56)$, and this may indicate a function distinct from that mediated by a secreted protein.

Our favorite hypothesis to explain a cytoprotective role for apoJ in autoimmune disease is that it aids in the disposal of bioactive cell debris and immune complexes. Consistent with this hypothesis, mRNA expression of gp330/megalin, a member of the LDL receptor superfamily, is induced in a subset of infiltrating cells present in myocarditic hearts (L. McLaughlin, unpublished). Gp330/megalin has been shown to bind and internalize apoJ through receptormediated endocytosis for subsequent degradation (57). A similarly supportive observation is the presence of reduced serum apoJ in patients with systemic lupus erythematosus (58). It is also possible that apoJ inhibits activation of complement initiated by immune complexes at the myocyte cell surface, which decelerates immune effector cell recruitment and activation. While FVB/N mice are genetically unable to secrete complement C5 $(59,60)$ required for assembly of a functional membrane attack complex known to be inhibited by apoJ, the protective action of apoJ is the result of interaction with IgG- and IgMantigen complexes, C1q and C3 $(34,61,62)$.

Our results suggest that apoJ limits the progression of autoimmune myocarditis, preventing long-term myocardial damage. Similar to the outcome for wildtype mice, human myocarditis frequently resolves over time, with little or no long-term consequences in heart function. However, in severe cases, extensive lymphocytic infiltration and myocyte necrosis can lead to hemodynamic deterioration (63). It will thus be important to determine if apoJ plays a similarly protective role against myocarditis in humans in the suppression of inflammatory damage.

\section{Acknowledgments}

We thank Cathy Saalfeld and Alicia Emley for help with the in situ hybridization assays and photography, and William S. Helton and Judith Bean for biostatistics. This work was supported by American Heart Association grants SW-96-24-F (L. McLaughlin) and
SW-96-43-S (D.P. Witte) and by National Institute of Environmental Health Sciences grant 08822 (J.A.K. Harmony and B.J. Aronow).

1. Cooper, G.S., Germolec, D., Heindel, J., and Selgrade, M. 1999. Linking environmental agents and autoimmune diseases. Environ. Health Perspect. 107:659-660.

2. Friman, G. 1999. The incidence and epidemiology of myocarditis. Eur. Heart J. 20:1063-1066.

3. Neumann, D.A., Burek, C.L., Baughman, K.L., Rose, N.R., and Herskowitz, A. 1990. Circulating heart-reactive antibodies in patients with myocarditis or cardiomyopathy. J. Am. Coll. Cardiol. 16:839-846.

4. Maisch, B., et al. 1985. Immunological cellular regulator and effector mechanisms in myocarditis. Herz. 10:8-14.

5. Lee, K.J., et al. 1999. Clinical outcomes of acute myocarditis in childhood. Heart. 82:226-233.

6. Huber, S.A. 1997. Autoimmunity in myocarditis: relevance of animal models. Clin. Immunol. Immunopathol. 83:93-102.

7. Finkel, M.S., et al. 1992. Negative inotropic effects of cytokines on the heart mediated by nitric oxide. Science. 257:387-389.

8. Hiraoka, Y., et al. 1993. Role of oxygen derived free radicals in the pathogenesis of Coxsackievirus B3 myocarditis in mice. Cardiovasc. Res. 27:957-961.

9. Kuan, A.P., et al. 1999. Genetic control of autoimmune myocarditis mediated by myosin-specific antibodies [ erratum 1999, 49:595-596]. Immunogenetics. 49:79-85.

10. Neu, N., Ploier, B., and Ofner, C. 1990. Cardiac myosin-induced myocarditis. Heart autoantibodies are not involved in the induction of the disease. J. Immunol. 145:4094-4100.

11. Huber, S.A. 1997. Coxsackievirus-induced myocarditis is dependent on distinct immunopathogenic responses in different strains of mice. Lab. Invest. 76:691-701.

12. Neu, N., et al. 1987. Cardiac myosin induces myocarditis in genetically predisposed mice. J. Immunol. 139:3630-3636.

13. Smith, S.C., and Allen, P.M. 1992. Neutralization of endogenous tumor necrosis factor ameliorates the severity of myosin-induced myocarditis. Circ. Res. 70:856-863.

14. Pummerer, C., Grassl, G., and Neu, N. 1995. Cellular immune mechanisms in myosin-induced myocarditis. Eur. Heart J. 16(Suppl. O):71-74.

15. Pummerer, C.L., et al. 1996. Cardiac myosin-induced myocarditis: target recognition by autoreactive $T$ cells requires prior activation of cardiac interstitial cells. Lab. Invest. 74:845-852.

16. Penninger, J.M., et al. 1993. The induction of experimental autoimmune myocarditis in mice lacking CD4 or CD8 molecules [erratum 1994, 179:371]. J. Exp. Med. 178:1837-1842.

17. Bachmaier, K., et al. 1996. Induction of autoimmunity in the absence of CD28 costimulation. J. Immunol. 157:1752-1757.

18. Kishimoto, C., Kitazawa, M., and Takada, H. 1998. Interstitial fibrinfibronectin deposition with $\mathrm{T}$ cell infiltrates precedes fibrosis in murine viral myocarditis. Int. J. Exp. Pathol. 79:417-423.

19. Gebhard, J.R., et al. 1998. Coxsackievirus B3-induced myocarditis: perforin exacerbates disease, but plays no detectable role in virus clearance. Am. J. Pathol. 153:417-428.

20. Yang, D., et al. 1999. Viral myocarditis: identification of five differentially expressed genes in coxsackievirus B3-infected mouse heart. Circ. Res. 84:704-712.

21. Swertfeger, D.K., Witte, D.P., Stuart, W.D., Rockman, H.A., and Harmony, J.A. 1996. Apolipoprotein J/clusterin induction in myocarditis: a localized response gene to myocardial injury. Am. J. Pathol. 148:1971-1983.

22. Jenne, D.E., and Tschopp, J. 1992. Clusterin: the intriguing guises of a widely expressed glycoprotein. Trends Biochem. Sci. 17:154-159.

23. Jordan-Starck, T.C., et al. 1994. Mouse apolipoprotein J: characterization of a gene implicated in atherosclerosis. J. Lipid Res. 35:194-210.

24. Rosenberg, M.E., and Silkensen, J. 1995. Clusterin: physiologic and pathophysiologic considerations. Int. J. Biochem. Cell Biol. 27:633-645.

25. Aronow, B.J., Lund, S.D., Brown, T.L., Harmony, J.A., and Witte, D.P. 1993. Apolipoprotein J expression at fluid-tissue interfaces: potential role in barrier cytoprotection. Proc. Natl. Acad. Sci. USA. 90:725-729.

26. Silkensen, J.R., et al. 1998. Temporal induction of clusterin in the periinfarct zone after experimental myocardial infarction in the rat. J. Lab. Clin. Med. 131:28-35.

27. Sawczuk, I.S., Hoke, G., Olsson, C.A., Connor, J., and Buttyan, R. 1989. Gene expression in response to acute unilateral ureteral obstruction. Kidney Int. 35:1315-1319.

28. Connor, J., et al. 1991. SGP-2 expression as a genetic marker of progressive cellular pathology in experimental hydronephrosis. Kidney Int. 39:1098-1103.

29. May, P.C., et al. 1992. Sulfated glycoprotein-2 expression increases in rodent brain after transient global ischemia. Brain Res. Mol. Brain Res. 15:33-39. 
30. Rosenberg, M.E., and Paller, M.S. 1991. Differential gene expression in the recovery from ischemic renal injury. Kidney Int. 39:1156-1161.

31. Vakeva, A., Laurila, P., and Meri, S. 1993. Co-deposition of clusterin with the complement membrane attack complex in myocardial infarction. Immunology. 80:177-182.

32. Wu, E., Brosnan, C.F., and Raine, C.S. 1993. SP-40,40 immunoreactivity in inflammatory CNS lesions displaying astrocyte/oligodendrocyte interactions. J. Neuropathol. Exp. Neurol. 52:129-134.

33. Polihronis, M., Paizis, K., Carter, G., Sedal, L., and Murphy, B. 1993. Elevation of human cerebrospinal fluid clusterin concentration is associated with acute neuropathology. J. Neurol. Sci. 115:230-233.

34. Hochgrebe, T.T., Humphreys, D., Wilson, M.R., and Easterbrook-Smith, S.B. 1999. A reexamination of the role of clusterin as a complement regulator. Exp. Cell Res. 249:13-21.

35. Shull, M.M., et al. 1992. Targeted disruption of the mouse transforming growth factor-beta 1 gene results in multifocal inflammatory disease. Nature. 359:693-699.

36. Shiverick, K.T., Thomas, L.L., and Alpert, N.R. 1975. Purification of cardiac myosin. Application to hypertrophied myocardium. Biochim. Biophys. Acta. 393:124-133.

37. Witte, D.P., Welch, T.R., and Beischel, L.S. 1991. Detection and cellular localization of human $\mathrm{C} 4$ gene expression in the renal tubular epithelial cells and other extrahepatic epithelial sources. Am. J. Pathol. 139:717-724.

38. Sylvester, S.R., Skinner, M.K., and Griswold, M.D. 1984. A sulfated glycoprotein synthesized by Sertoli cells and by epididymal cells is a component of the sperm membrane. Biol. Reprod. 31:1087-1101.

39. Perks, C.M., Newcomb, P.V., Norman, M.R., and Holly, J.M. 1999. Effect of insulin-like growth factor binding protein-1 on integrin signalling and the induction of apoptosis in human breast cancer cells. J. Mol. Endocrinol. 22:141-150.

40. Laemmli, U.K. 1970. Cleavage of structural proteins during the assembly of the head of bacteriophage T4. Nature. 227:680-685.

41. Kofler, H., Kofler, R., Wolf, H., Muller, P.U., and Wick, G. 1984. Development of an enzyme-linked immunosorbent assay for the detection of autoantibodies against thyroglobulin in chickens. J. Immunol. Methods. 69:243-252.

42. Sussman, M.A., et al. 1998. Myofibril degeneration caused by tropomodulin overexpression leads to dilated cardiomyopathy in juvenile mice. J. Clin. Invest. 101:51-61.

43. Swertfeger, D.K. 1997. Role of apolipoprotein J in autoimmune myocarditis. Ph.D. thesis. University of Cincinnati. Cincinnati, Ohio, USA. 143 pp.

44. Kapadia, S.R. 1999. Cytokines and heart failure. Cardiol. Rev. 7:196-206.

45. Kubota, T., et al. 1997. Dilated cardiomyopathy in transgenic mice with cardiac-specific overexpression of tumor necrosis factor-alpha. Circ. Res. 81:627-635.

46. Bachmaier, K., et al. 1997. Low-molecular-weight tumor necrosis factor receptor p55 controls induction of autoimmune heart disease. Circulation. 95:655-661.

47. Huber, S.A., and Pfaeffle, B. 1994. Differential Th1 and Th2 cell respons- es in male and female BALB/c mice infected with coxsackievirus group B type 3. J. Virol. 68:5126-5132.

48. Dalal, M., Kim, S., and Voskuhl, R.R. 1997. Testosterone therapy ameliorates experimental autoimmune encephalomyelitis and induces a $\mathrm{T}$ helper 2 bias in the autoantigen-specific T lymphocyte response. $J$. Immunol. 159:3-6.

49. Huber, S.A., Job, L.P., and Woodruff, J.F. 1981. Sex-related differences in the pattern of coxsackievirus B-3-induced immune spleen cell cytotoxicity against virus-infected myofibers. Infect. Immun. 32:68-73.

50. Gauntt, C.J. 1997. Roles of the humoral response in coxsackievirus Binduced disease. Curr. Top. Microbiol. Immunol. 223:259-282.

51. Latif, N., Zhang, H., Archard, L.C., Yacoub, M.H., and Dunn, M.J. 1999. Characterization of anti-heart antibodies in mice after infection with coxsackie B3 virus. Clin. Immunol. 91:90-98.

52. Sintich, S.M., et al. 1999. Cytotoxic sensitivity to tumor necrosis factoralpha in PC3 and LNCaP prostatic cancer cells is regulated by extracellular levels of SGP-2 (clusterin). Prostate. 39:87-93.

53. Sensibar, J.A., et al. 1995. Prevention of cell death induced by tumor necrosis factor alpha in LNCaP cells by overexpression of sulfated glycoprotein-2 (clusterin). Cancer Res. 55:2431-2437.

54. Humphreys, D., Hochgrebe, T.T., Easterbrook-Smith, S.B., Tenniswood, M.P., and Wilson, M.R. 1997. Effects of clusterin overexpression on TNFalpha- and TGFbeta-mediated death of L929 cells. Biochemistry. 36:15233-15243.

55. Jin, G., and Howe, P.H. 1997. Regulation of clusterin gene expression by transforming growth factor beta. J. Biol. Chem. 272:26620-26626.

56. Yang, C.R., et al. 1999. Isolation of Ku70-binding proteins (KUBs). Nucleic Acids Res. 27:2165-2174.

57. Kounnas, M.Z., et al. 1995. Identification of glycoprotein 330 as an endocytic receptor for apolipoprotein J/clusterin [erratum 1995, 270:23234]. J. Biol. Chem. 270:13070-13075.

58. Newkirk, M.M., Apostolakos, P., Neville, C., and Fortin, P.R. 1999. Systemic lupus erythematosus, a disease associated with low levels of clusterin/apoJ, an antiinflammatory protein. J. Rheumatol. 26:597-603.

59. Jenne, D.E., and Tschopp, J. 1989. Molecular structure and functional characterization of a human complement cytolysis inhibitor found in blood and seminal plasma: identity to sulfated glycoprotein 2, a constituent of rat testis fluid. Proc. Natl. Acad. Sci. USA. 86:7123-7127.

60. Choi, N.H., Mazda, T., and Tomita, M. 1989. A serum protein SP40,40 modulates the formation of membrane attack complex of complement on erythrocytes. Mol. Immunol. 26:835-840.

61. Sawtell, N.M., Hartman, A.L., Weiss, M.A., Pesce, A.J., and Michael, J.G. 1988. C3 dependent, C5 independent immune complex glomerulopathy in the mouse. Lab. Invest. 58:287-293.

62. Roeth, P.J., and Easterbrook-Smith, S.B. 1996. C1q is a nucleotide binding protein and is responsible for the ability of clusterin preparations to promote immune complex formation. Biochim. Biophys. Acta. 1297:159-166.

63. Barry, W.H. 1994. Mechanisms of immune-mediated myocyte injury. Circulation. 89:2421-2432. 